\title{
Field exploration: when to start extracting?
}

\author{
N. Langrené ${ }^{\mathrm{a}}$, W. Chen ${ }^{\mathrm{a}}, \mathrm{Z}$. Zhu ${ }^{\mathrm{a}}$ \\ ${ }^{a}$ CSIRO DATA61, Docklands, VIC, 3008, Australia \\ Email: Nicolas.Langrene@csiro.au
}

\begin{abstract}
For mining companies, mineral exploration processes such as drilling holes are very costly, but are necessary to have more accurate assessment of the potential quality and quantity of certain minerals in a new field before mine development and extraction can start. Extended exploration periods can drastically reduce geological uncertainties but can be prohibitively expensive. By contrast, insufficient exploration process will leave substantial uncertainty in the assessment of ore grade and reserve in the field. For a mining company, choosing the optimal time to stop the exploration process and start mine extraction can significantly add value to a new mining project. Due to the large uncertainties present, adopting dynamic and reactive strategies in the decision-making process can mean the difference between a profitable and a lossmaking mining project.

In this paper, we present a mathematical approach for solving the exploration-to-extraction timing problem. More specifically, we model the decision-making process of a mining company which holds a lease to explore a selected land for a limited time, before exercising the option to start a mining operation or cease the lease. This exploration-to-extraction decision-making process is largely determined by two main sources of uncertainties: the current estimated mineral reserve and the future ore price. Mathematically, we formulate this problem as an optimal stopping problem. The decision to start mining or not is chosen so as to maximise the probability that the whole mining project, after deducting exploration costs, would meet a predefined minimum profit target.

In order to solve this mathematical formulation of the exploration-to-extraction problem, we use the simulation-based Regression Monte Carlo method. We assume the mineral ore price follows a mean-reverting process with jumps and the estimated reserve follows a pure jump process with decreasing variance as long as exploration occurs, to account for the uncertainty reduction provided by exploration. We present the output results from the model in the form of intuitive graphical displays, in particular the optimal decisions over time as a function of the two uncertainties. The objective of this paper is to illustrate the feasibility and benefit of using such a mathematical approach for optimally timing the exploration process, and to contribute to the efficient management of mining projects by adopting such innovative decision support tools.
\end{abstract}

Keywords: Mining, exploration, optimal stopping, reserve estimation, uncertainty, real option, project valuation 


\section{INTRODUCTION}

We consider the decision-making process of a mining company which holds a lease to explore a piece of land for a limited time, before exercising the option to start a mining operation. The purpose of the exploration process is to establish the recoverable reserve in the field through hole drilling operations. Before initiating extraction operations, the company needs to ensure, with a high degree of confidence, that the recoverable reserve is above a minimum profitability threshold. In addition to the reserve level, the second main source of uncertainty is the mineral price in the market. The future mineral price directly impacts the profitability prospect of the future mining project, and therefore is a major factor influencing the decision to continue or terminate the current exploration.

In the literature, only a few of publications analyse the exploration process under price and reserve uncertainty as a real option problem. Paddock et al. [1988] apply the real option approach to an offshore petroleum lease under random quantity of recoverable hydrocarbon. In Armstrong et al. [2004], the option to acquire additional information (drilling more wells) is valued by using Bayesian techniques and copulas to update the estimated prospects when new information is gathered. For application in mining, the most relevant paper is Cortazar et al. [2001], which treats the exploration process as a real option under both price and geological uncertainties. These two uncertainties are modeled by simple Geometric Brownian Motion (GBM) processes, and the state variable vector is further reduced to one single state variable, namely the product of the two GBM variables. At the end of the exploration period, the value of the mine is given by the real option valuation from Brennan and Schwartz [1985], for which the mine manager has the operational flexibility to temporarily shut down the mine in response to commodity prices. The combination of exploration and extraction is neatly formulated in Cortazar et al. [2001] as a compound real option. In this paper, we focus on the time period of the exploration process. We use the probability that the future mine reaches a profitability target as the objective function, and we maximise this probability dynamically. The output from this algorithm can tell us when to stop exploration, and whether to start the extraction phase or to abandon the lease, by using as inputs the current mineral market price and the estimated reserve.

The paper is organized as follows. Section 2 provides the mathematical formulation of this exploration problem. Section 3 describes the regression Monte Carlo method used to solve the problem. Section 4 provides the numerical results and sensitivity analysis, as well as displays of the optimal decision regions of the examples. Finally, Section 5 provides our conclusions.

\section{Problem FORMUlation}

In this section, we describe the mathematical model chosen for the estimated reserve dynamics (subsection 2.1), the metal price dynamics (subsection 2.2) and the objective function (subsection 2.3).

\subsection{Estimated reserve}

Let $T$ be the term of the exploration lease, and let $\Pi=\left\{t_{0}=0<t_{1}<t_{2}<\ldots<t_{N}=T\right\}$ be the list of time to make the decision of stopping exploration and either start extracting or abandoning the field. The stopping time when this decision occurs is denote by $\tau \in \Pi$, and the estimated reserve at time $t_{i}$ is denoted by $R_{t_{i}}$. While exploration is occurring $\left(t_{i+1} \leq \tau\right)$, we model the dynamics of $R$ by:

$$
R_{t_{i+1}}=R_{t_{i}} \times J_{R}\left(t_{i}\right)
$$

where $J_{R}\left(t_{i}\right)=e^{\phi_{R}\left(t_{i}\right)}, \phi_{R}\left(t_{i}\right) \sim \mathcal{N}\left(-\frac{\sigma_{J_{R}}^{2}\left(t_{i}\right)}{2}, \sigma_{J_{R}}^{2}\left(t_{i}\right)\right)$ is a jump component with mean $\mathbb{E}\left[J_{R}\left(t_{i}\right)\right]=1$, and

$$
\sigma_{J_{R}}\left(t_{i+1}\right)=\sigma_{J_{R}}\left(t_{i}\right) \times \lambda
$$

where $0<\lambda<1$ is a constant. This volatility model means that the amplitude of the jumps decreases over time, thus the reserve estimate improves.

Without the exploration time limit $T$, the estimated reserve would eventually converge to the true reserve $R_{\infty}$. At time $\tau$, the best estimate of the reserve distribution is given by the conditional distribution $R_{\infty} \mid R_{\tau}$, given explicitly in Appendix A. After $\tau$, we assume for simplicity that the reserve estimate remains the same, in other words, we assume that the reserve information can only be improved during exploration. 


\subsection{Metal price}

Let $S=\left(S_{t}\right)_{0 \leq t \leq T}$ be the metal price. We assume $S$ follows mean-reverting positive dynamics of the type:

$$
d S_{t}=\kappa_{S}\left(\bar{S}-S_{t}\right) d t+\sigma_{S} S_{t} d W_{S}(t)+\left(J_{S}-1\right) S_{t} d P_{S}(t), t \geq 0
$$

where $W=\left(W_{S}(t)\right)_{0 \leq t \leq T}$ is a Brownian motion, $J_{S}=e^{\phi_{S}}, \phi_{S} \sim \mathcal{N}\left(-\frac{\sigma_{J_{S}}^{2}}{2}, \sigma_{J_{S}}^{2}\right)$ is a jump component with mean $\mathbb{E}\left[J_{S}\right]=1$, which occurs at random Poisson times $d P_{S}(t)=\left\{\begin{array}{ll}1 & \text { with prob. } \lambda_{S} d t \\ 0 & \text { with prob. } 1-\lambda_{S} d t\end{array}\right.$. The parameter $\kappa_{S}$ is the mean reversion speed to the constant level $\bar{S}$ and $\sigma_{S}$ is the constant volatility. $S$ and $R$ are assumed to be independent processes.

\subsection{Objective function}

Recall that $\tau$ is the stopping time when exploration stops and a decision is made on starting extraction or abandoning the field. Denote the decision outcome by $\gamma \in\{0,1\}$, where $\gamma=0$ means abandon the field, and $\gamma=1$ means proceed with the mine construction and start extraction.

Before $\tau$, each time period of exploration incurs a fixed cost $K_{e}>0$. The accumulated total exploration cost up to time $\tau$ (discounted to time 0 ) is given by:

$$
E C_{\tau}=\sum_{0 \leq t_{i}<\tau} D F_{t_{i}} \times K_{e}
$$

where $D F_{t_{i}}$ is the discount factor at time $t_{i}$.

At time $\tau, \gamma=0$ or 1 . If $\gamma=0$ the field is abandoned, thus no other cash-flow occurs. If $\gamma=1$, we assume there is a fixed cost $K_{s}$ for setting up the mine. Define $S C_{\tau}=D F_{\tau} \times K_{s}$ as this starting cost, discounted to time 0 .

Finally, after time $\tau$, when $\gamma=1$, the (random) future cash-flow of the mine at time $t_{i} \geq \tau$ is given by:

$\left(q \times S_{t_{i}}-A_{t_{i}}\right) \Delta t$, if the mine is not depleted at time $t_{i}$

0 otherwise

where:

- $S_{t_{i}}$ is the metal price at time $t_{i}$ (equation (3)).

- $q \times \Delta t$ is the amount of metal extracted during one time period of length $\Delta t$ (the production rate $q$ is assumed to be constant).

- $A_{t_{i}} \times \Delta t$ is the total operational cost during one time period $\Delta t$. We assume that $A_{t_{i}}=A_{0} \times e^{r_{\text {inf }} t_{i}}$ where the initial cost $A_{0}$ is constant and $r_{\text {inf }}$ is the inflation rate.

The sum of all these future cash-flows, discounted to time $\tau$, is given by

$$
\mathrm{CF}_{\tau}=\left(\sum_{\tau<t_{i} \leq \tau+\left\lfloor\frac{R_{\infty}}{q}\right\rfloor \Delta t} D F_{t_{i}} \times\left(q \times S_{t_{i}}-A_{t_{i}}\right) \Delta t\right)
$$

Indeed, because the actual total reserve before extraction is $R_{\infty}$, the number of future cash-flow periods is equal to $\left\lfloor R_{\infty} / q\right\rfloor$, after which the mine will be depleted. For simplicity, we do not extract the final incomplete period which occurs when $R_{\infty} / q>\left\lfloor R_{\infty} / q\right\rfloor$.

Finally, we define the objective function of the decision-maker as follows:

$$
V_{t}=V_{t}\left(S_{t}, R_{t}\right)=\left\{\max _{\tau \in \Pi \cap[t, T]} \mathbb{P}\left[-E C_{\tau}-S C_{\tau}+\mathrm{CF}_{\tau} \geq B_{\tau} \mid S_{t}, R_{t}\right]-p\right\}_{+}, t \in \Pi
$$


where $B_{t_{i}}:=D F_{T_{i}} \times B$, for a lower profit target $B$ and a desired probability $p$ of meeting this target chosen by the mining company. There is a trade-off between $B$ and $p$ : setting a high profit target $B$ would mean that timing decisions in an optimal manner $\tau^{*}$ might not be sufficient to meet the probability requirement $p$. Conversely, setting a low profit target $B$ would mean that meeting the probability requirement would be easy, but also that some profit potential might have been lost. For additional discussion of this type of objective function, we refer to Zhang et al. [2016].

The positive cap $\{x\}_{+}=\max \{x, 0\}$ is due to the option to abandon the field: when the maximum probability $\max _{\tau \in \Pi \cap[t, T]} \mathbb{P}\left(-E C_{\tau}-S C_{\tau}+\mathrm{CF}_{\tau} \geq B_{\tau} \mid S_{t}, R_{t}\right)$ is still lower than the requested minimum probability target $p$, it is better not to build the mine and abandon this field. Remark finally that the exploration cost $E C$ is taken into account, so that the net profit is used to assess the objective.

The objective formulation (5) has strong advantages over classical expected discounted Net Price Value (see Zhang et al. [2016]). The decision policy resulting from (5) ensures that the profitability target will be met with a high probability. As only one path of price and recoverable reserve will actually happen in the future, the criteria (5) provides the highest chance that the profitability target will actually be met in reality. By contrast, expected NPV and even expected utility of NPV leave the possibility of very negative scenarios to be averaged out by the possibility of very positive scenarios. This results in a very unpredictable actual profit or loss of the future mine, the theoretical highest ex-ante expectation being of little help due to the remaining variance around it. The formulation (5) solves the problem by improving predictability and being more robust to extreme scenarios. Moreover, the two target parameters $B$ and $p$ make the residual bet on the profitability of the mining project clear and transparent.

\section{NUMERICAL METHOD}

To solve equation (5), we first compute the inner probability

$$
\mathcal{P}_{t}:=\max _{\tau \in \Pi \cap[t, T]} \mathbb{P}\left(-E C_{\tau}-S C_{\tau}+\mathrm{CF}_{\tau} \geq B_{\tau} \mid S_{t}, R_{t}\right)
$$

by dynamic programming, then we compute the decision outcomes

$$
\gamma_{t}=\mathbb{1}\left\{\mathcal{P}_{t}>p\right\}
$$

to finally obtain $V_{t}$ :

$$
V_{t}=\left(\mathcal{P}_{t}-p\right) \mathbb{1}\left\{\gamma_{t}\right\}
$$

To obtain $\mathcal{P}_{t}$, we compute the optimal stopping time $\tau^{*}$ by backward induction. Recall that the exploration lease expires at time $T$, so that the decision about whether to start building a mine or not must be made before $T(\tau \leq T)$. Consequently, at time $t_{N}=T$ :

$$
\tau_{T}^{*}=\tau_{T}^{*}\left(S_{T}, R_{T}\right)=T
$$

Then, for each $t_{i}<t_{N}$ the Bellman induction principle reads:

$$
\tau_{t_{i}}^{*}=\tau_{t_{i}}^{*}\left(S_{t_{i}}, R_{t_{i}}\right)=\underset{t \in\left\{t_{i}, \tau_{t_{i+1}}^{*}\right.}{\arg \max } \mathbb{P}\left(-E C_{t}-S C_{t}+\mathrm{CF}_{t} \geq B_{t} \mid S_{t_{i}}, R_{t_{i}}\right)
$$

To compute this optimal stopping time in practice, we first condition analytically with respect to $R_{t_{i}}$ (see Appendix B). Then, we compute the remaining conditional expectations with respect to $S_{t_{i}}$ using Longstaff and Schwartz [2001]'s implementation of the Least-Squares Monte Carlo algorithm (regression on the realized paths under optimal future decisions). More precisely, we use the two-stage semi-analytic regression proposed in Zhang et al. [2016], which is particularly well suited to discontinuous responses such as the $\{0,1\}$ responses involved in the probability objective (7).

\section{NUMERicAl RESULTS}

In this section, we solve the problem (5) on a numerical example. We set the numerical parameters to the values similar to the example in Brennan and Schwartz [1985]: $S_{0}=0.5, A_{0}=0.5, R_{0}=150, q=10$, $r=10 \%, r_{\text {inf }}=0.08$. For the additional parameters in the dynamics of $S$ and $R$, we set $\kappa_{S}=0.1, \bar{S}=S_{0}$, 
$t=2$

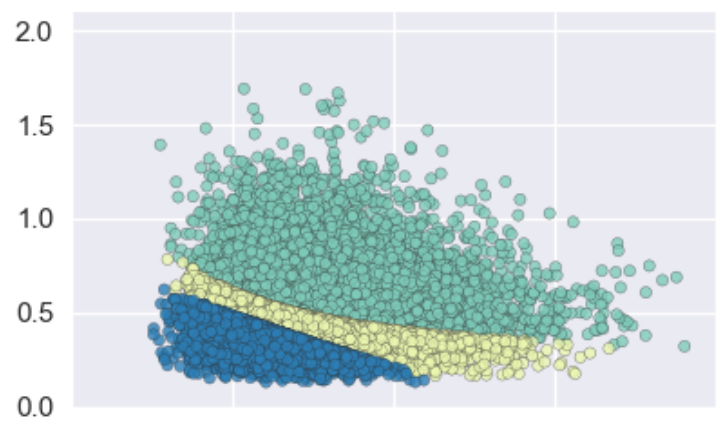

$t=6$

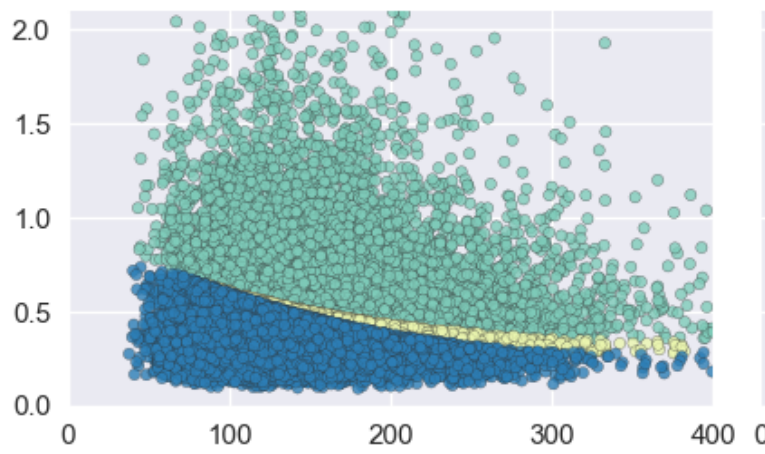

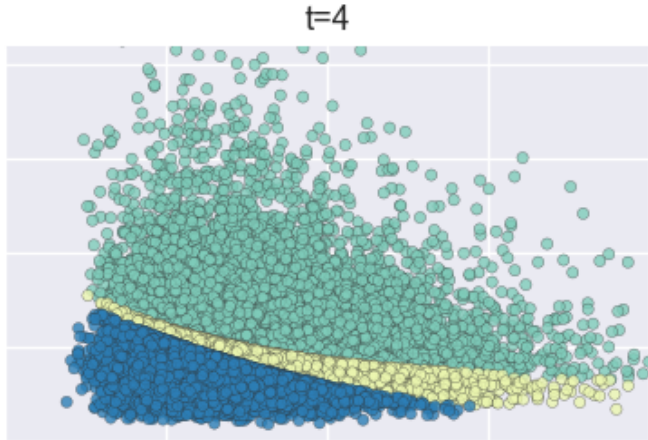

$t=8$

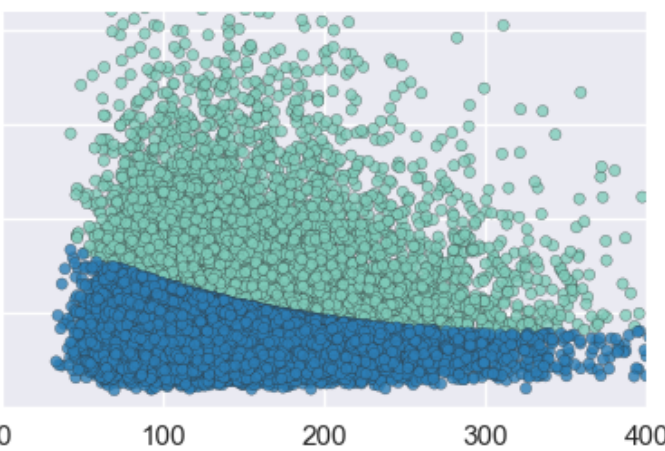

Figure 1. Optimal decision regions at different time $t$ ( $\mathrm{x}$-axis is reserve, $\mathrm{y}$-axis is price)
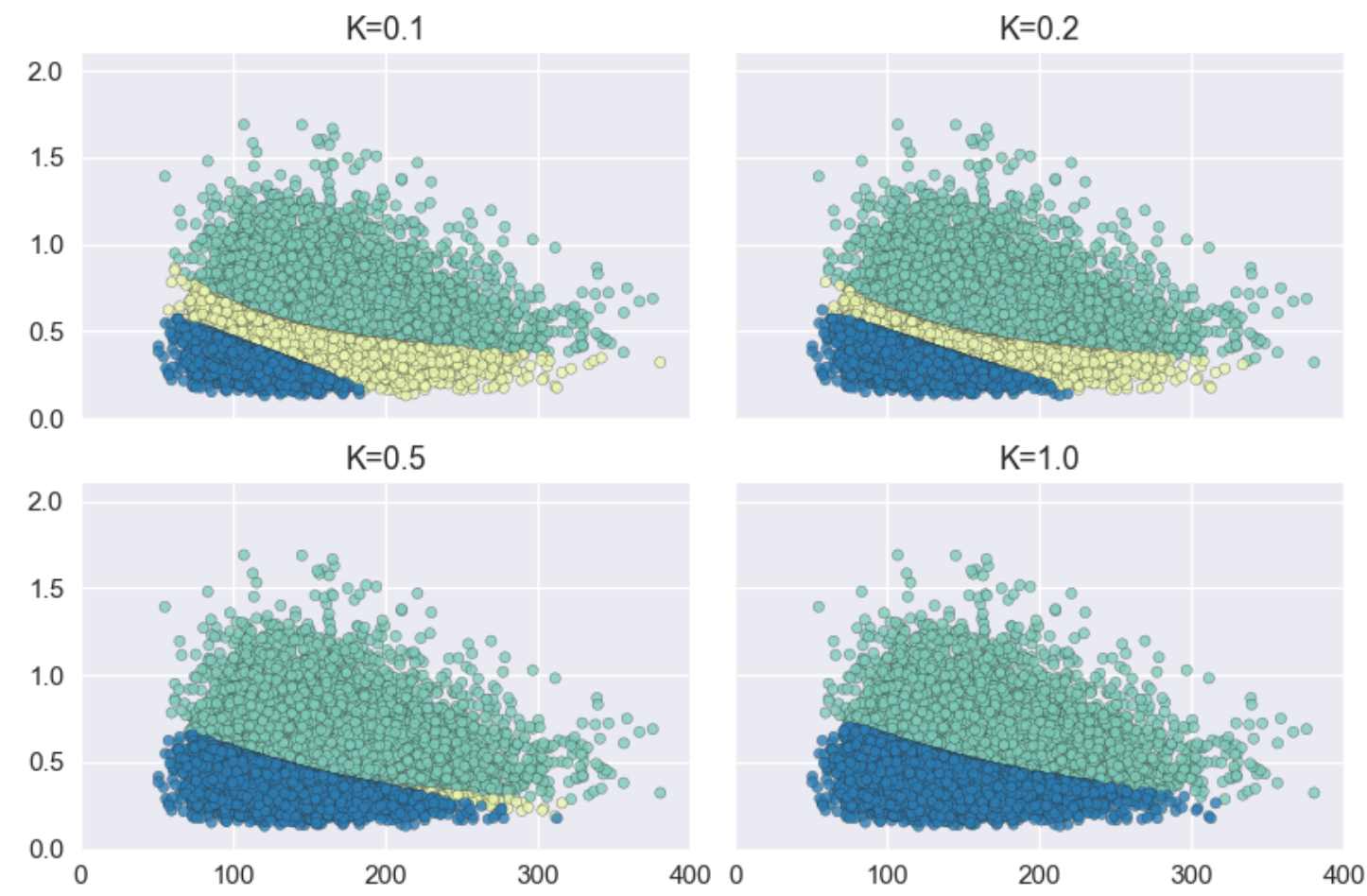

Figure 2. Optimal decision regions for different exploration cost $K$ 
$\sigma_{S}=0.28, \lambda_{S}=0.05, \sigma_{J_{S}}=0.05, \sigma_{J_{R}}(0)=0.15, \lambda=0.925$. For the additional parameters in the cashflows and the objective function, we set $K_{e}=0.2, K_{s}=10, B=10, p=40 \%$. We choose a constant time mesh $t_{i+1}-t_{i}=0.5$ (two decision times per year) and $T=10$ years.

Figure 1 displays the optimal exploration decisions at different decision time on the Monte Carlo dataset. The optimal decision also depends on the estimated reserve (x-axis) and the current marekt price (y-axis). The green region at the top is the region where the optimal decision is to stop exploration and start building the mine. The yellow region in the middle is the region where it is optimal to continue exploring before making any other decision. Finally, the bottom blue region is where the optimal decision is to stop exploration and abandon the field. This means the best exploration stopping time is unable to generate a profitability success rate higher than the predefined target $p$.

Unsurprisingly, the green region (start extraction) covers the top right corner (high price, high expected reserve), while the blue region (abandon) covers the bottom left corner (low price, low expected reserve). The yellow band (keep exploring) stands in between these two regions.

One can see in Figure 1 that the main change over time is that the yellow band shrinks and eventually disappears around the eighth year $(t=8)$, two years before the end of the lease $(T=10)$. This illustrates how the value of waiting to gather more information disappears over time once the deadline $T$ approaches. Several parameters directly affect the relative size of these decisions regions. For example, Figure 2 illustrates the effect of the exploration cost $K_{e}$. As expected, increasing $K_{e}$ extends the blue region, so much so that the yellow region completely disappears when $K_{e}=1.0$. Increasing other parameters such as the probability target $p$ or the profit target $B$ has a similar effect on the optimal decision regions.

\section{Conclusion}

This paper focuses on the exploration period before the start of natural resource extraction projects. The value of gathering more information through drilling before developing a mine is accounted for using the real option approach, combined with a model of recoverable reserve for which the uncertainty of the reserve estimate decreases during exploration. We use Regression Monte Carlo techniques to solve the problem numerically and compute the optimal time to stop exploration, as a function of the observable price and reserve estimate, so as to maximize the probability for the future mining project to be profitable.

We display the optimal decision regions over time until the end of the exploration lease. Depending on the level of price and reserve, the optimal decision can be to stop exploration and start extraction (high price, high reserve), to stop exploration and abandon the field (low price, low reserve), or to keep exploring (intermediate band). The location of the waiting band depends on many other factors, such as exploration costs and the profitability target.

One area for further development of this research is the valuation of the mine after the end of the exploration period. In this paper we simply assume steady extraction without interruption until depletion, while in reality extraction itself offers various management option which can add value to the mining project (Savolainen [2016]). In the lines of Cortazar et al. [2001], real option techniques could be used for the extraction period first (cf. Chen et al. [2016] for example) and then compounded with the options available during the exploration period as done in this paper, though such a combination of options is likely to introduce computational challenges. Another area of extension is the choice of input parameters and further analysis of their effect on optimal decisions and the project valuation.

ApPendix A Distribution of $R_{\infty} \mid R_{t_{m}}$

Let $t_{n}>t_{m}$. Using equation (1):

$$
R_{t_{n}}=R_{t_{m}} \Pi_{j=m}^{n-1} J_{R}\left(t_{j}\right)=R_{t_{m}} e^{\sum_{j=m}^{n-1} \phi_{R}\left(t_{j}\right)}
$$

Now, $\sum_{j=m}^{n-1} \phi_{R}\left(t_{j}\right)$ is a Gaussian variable with mean $-\frac{1}{2} \sum_{j=m}^{n-1} \sigma_{J_{R}}^{2}\left(t_{j}\right)$ and variance $\sum_{j=m}^{n-1} \sigma_{J_{R}}^{2}\left(t_{j}\right)$. From equation (2), $\sigma_{J_{R}}\left(t_{i}\right)=\sigma_{0} \lambda^{j}$, and therefore

$$
\sum_{j=m}^{n-1} \sigma_{J_{R}}^{2}\left(t_{j}\right)=\sigma_{0}^{2} \frac{\lambda^{2 m}-\lambda^{2 n}}{1-\lambda^{2}}
$$

To sum up, the distribution of $R_{t_{n}} \mid R_{t_{m}}$ is lognormal with mean $R_{t_{m}}$ and variance $R_{t_{m}}^{2}\left(\exp \left(\sigma_{0}^{2} \frac{\lambda^{2 m}-\lambda^{2 n}}{1-\lambda^{2}}\right)-1\right)$ 
In particular, the distribution of $R_{\infty} \mid R_{t_{m}}$ is lognormal with mean $R_{t_{m}}$ and variance $R_{t_{m}}^{2}\left(\exp \left(\sigma_{0}^{2} \frac{\lambda^{2 m}}{1-\lambda^{2}}\right)-1\right)$.

\section{APPENDIX B RESERVE CONDITIONING}

Using the distribution from the previous appendix, one can recursively compute the inner probability (6) as follows. For every $t_{n} \geq t_{m} \geq 0$ :

$$
\begin{aligned}
& \mathbb{P}\left(-E C_{t_{n}}-S C_{t_{n}}+\mathrm{CF}_{t_{n}} \geq B_{t_{n}} \mid S_{t_{m}}, R_{t_{m}}\right) \\
= & \mathbb{P}\left(\sum_{t_{n}<t_{i} \leq t_{n}+\left\lfloor\frac{R_{\infty}}{q}\right\rfloor \Delta t} D F_{t_{i}} \times\left(q \times S_{t_{i}}-\kappa\right) \Delta t \geq \tilde{B}_{t_{n}} \mid S_{t_{m}}, R_{t_{m}}\right) \\
= & \sum_{t_{k} \geq t_{n}} \mathbb{P}\left(\sum_{t_{n}<t_{i} \leq t_{k}} D F_{t_{i}} \times\left(q \times S_{t_{i}}-\kappa\right) \Delta t \geq \tilde{B}_{t_{n}} \mid S_{t_{m}}\right) \mathbb{P}\left(t_{k}=t_{n}+\left\lfloor\frac{R_{\infty}}{q}\right\rfloor \Delta t \mid R_{t_{m}}\right)
\end{aligned}
$$

where $\tilde{B}_{t_{n}}:=B_{t_{n}}+E C_{t_{n}}+S C_{t_{n}}=B_{t_{n}}+\sum_{0 \leq t_{i}<t_{n}} D F_{t_{i}} \times K_{e}+D F_{t_{n}} \times K_{s}$ and where we used the independence between $S$ and $R$. Using the explicit distribution of $R_{\infty} \mid R_{t_{m}}$ derived in Appendix A:

$$
\begin{aligned}
\mathbb{P}\left(t_{k}=t_{n}+\left\lfloor\frac{R_{\infty}}{q}\right\rfloor \Delta t \mid R_{t_{m}}\right) & =\mathbb{P}\left(\frac{t_{k}-t_{n}}{\Delta t} \leq \frac{R_{\infty}}{q}<\frac{t_{k+1}-t_{n}}{\Delta t} \mid R_{t_{m}}\right) \\
& =\mathbb{P}\left(\log \left(\frac{q}{R_{m}} \frac{t_{k}-t_{n}}{\Delta t}\right) \leq \sum_{j=m}^{n-1} \phi_{R}\left(t_{j}\right)<\log \left(\frac{q}{R_{m}} \frac{t_{k+1}-t_{n}}{\Delta t}\right) \mid R_{t_{m}}\right) \\
& =p_{m, n, k+1}\left(R_{m}\right)-p_{m, n, k}\left(R_{m}\right)
\end{aligned}
$$

where

$$
p_{m, n, k}\left(R_{m}\right):=\Phi\left(\frac{1}{2} \lambda^{m} \frac{\sigma_{0}}{\sqrt{1-\lambda^{2}}}+\frac{1}{\lambda^{m} \frac{\sigma_{0}}{\sqrt{1-\lambda^{2}}}} \log \left(\frac{q}{R_{m}} \frac{t_{k}-t_{n}}{\Delta t}\right)\right)
$$

where $\Phi$ is the cumulative distribution function of a standard Gaussian variable.

\section{REFERENCES}

M. Armstrong, A. Galli, W. Bailey, and B. Couët. (2004). Incorporating technical uncertainty in real option valuation of oil projects. Journal of Petroleum Science and Engineering, 44(1-2):67-82.

M. Brennan and E. Schwartz. (1985). Evaluating natural resource investment. Journal of Business, 58(2):135-15.

W. Chen, N. Langrené, and T. Tarnopolskaya. (2016). Natural resource extraction with production target: the real option value of variable extraction rate. In ROC2016, 20th Real Option Conference.

G. Cortazar, E. Schwartz, and J. Casassus. (2001). Optimal exploration investments under price and geologicaltechnical uncertainty: a real options model. $R \& D$ Management, 31(2):181-189.

F. Longstaff and E. Schwartz. (2001). Valuing American options by simulation: a simple least-squares approach. Review of Financial Studies, 14(1):113-147.

J. Paddock, D. Siegel, and J. Smith. (1988). Option valuation of claims on real assets: the case of offshore petroleum leases. Quarterly Journal of Economics, 103(3):479-508.

J. Savolainen. (2016). Real options in metal mining project valuation: Review of literature. Resources Policy, 50: 49-65.

R. Zhang, N. Langrené, Y. Tian, Z. Zhu, F. Klebaner, and K. Hamza. (2016). Sharp target range strategies with application to dynamic portfolio selection. 